\title{
Large-Eddy Simulations and Damped-Oscillator Models of the Unsteady Ekman Boundary Layer*
}

\author{
Mostafa Momen And Elie Bou-Zeid \\ Department of Civil and Environmental Engineering, Princeton University, Princeton, New Jersey
}

(Manuscript received 4 February 2015, in final form 25 August 2015)

\begin{abstract}
The Ekman boundary layer (EBL) is a central problem in geophysical fluid dynamics that emerges when the pressure gradient force, the Coriolis force, and the frictional force interact in a flow. The unsteady version of the problem, which occurs when these forces are not in equilibrium, is solvable analytically only for a limited set of forcing variability regimes, and the resulting solutions are intricate and not always easy to interpret. In this paper, large-eddy simulations (LESs) of neutral atmospheric EBLs are conducted under various unsteady forcings to reveal the range of physical characteristics of the flow. Subsequently, it is demonstrated that the dynamics of the unsteady EBL can be reduced to a second-order ordinary differential equation that is very similar to the dynamical equation of a damped oscillator, such as a mass-spring-damper system. The validation of the proposed reduced model is performed by comparing its analytical solutions to LES results, revealing very good agreement. The reduced model can be solved for a wide range of variable forcing conditions, and this feature is exploited in the paper to elucidate the physical origin of the inertia (mass), energy storage (spring), and energy dissipation (damper) attributes of Ekman flows.
\end{abstract}

\section{Introduction}

The steady-state Ekman boundary layer (EBL) is a canonical flow of interest in a very wide range of applications. It results from the balance of the pressure gradient force (or wind stress for the upper ocean) driving the flow, the Coriolis force, and a drag force (viscous or turbulent); it is hence observed in various rotating flows, such as in the upper ocean or the lower atmosphere [the oceanic and atmospheric boundary layers (Vallis 2006)]. If the viscosity is assumed constant or if it has a simple functional dependence on the distance from the surface, the governing equations for the steady flow can be readily solved [as done, for example, by Ekman himself (Ekman 1905)]. More general steady-state cases (for example, under nonneutral static stabilities) have also been studied using experiments (Caldwell et al. 1972;

\footnotetext{
* Supplemental information related to this paper is available at the Journals Online website: http://dx.doi.org/10.1175/JAS-D-15-0038.s1.

Corresponding author address: Elie Bou-Zeid, E-414 E-Quad, Dept. of Civil and Environmental Engineering, Princeton University, 59 Olden Street, Princeton, NJ 08544.

E-mail: ebouzeid@princeton.edu
}

Sous et al. 2013) and numerical simulations (Shingai and Kawamura 2004; Spalart et al. 2008; Martlatt et al. 2010). For instance, steady Ekman flows with a gradually varying eddy viscosity were studied numerically and analytically by Miles (1994), Grisogono (1995), and Parmhed et al. (2005). Berger and Grisogono (1998) and Tan (2001) also examined the steady wind profiles with height-varying eddy viscosity and pressure gradient in a baroclinic EBL, deriving the time-invariant EBL solutions. Moreover, Veronis (1967) underlined the mathematical similarity between Ekman's homogeneous rotating steady-flow solution and Prandtl's solution for a thermally driven nonrotating slope flow. The obtained solutions help explain many important geophysical phenomena, such as Ekman pumping; however, the steady-state assumption limits the scope of these investigations.

Real-world boundary layer flows in the atmosphere and oceans are almost always changing in time $t$; their unsteady characteristics result from time variability in the horizontal pressure gradient force or in the frictional stress (Bonner and Paegle 1970). The complex manifestations of this variability make the analysis of unsteady EBLs challenging; however, their wide relevance (Hering and Borden 1962; Bonner and Paegle 1970) 
continues to make them the focus of numerous research efforts (Prandle 1982; Hsu et al. 2000; Lohmann et al. 2006; Gayen et al. 2010). These efforts often focus on numerical and experimental approaches, but obtaining analytical solutions for the unsteady case remains of high interest because of the insights they can yield into the dynamics of the flow. Most previous analytical studies have focused on unsteadiness emanating from the variability of the frictional stress caused by, for example, the sudden onset of stable stratification that reduces the eddy viscosity [this approach was first used by Blackadar (1957) to explain the nocturnal low-level jet]. Recent studies include Du and Rotunno (2014) and Du et al. (2015), who investigated nocturnal low-level jet formation with diurnally varying boundary layer winds. Nevertheless, unsteadiness resulting from the pressure forcing associated in the atmosphere with mesoscale variability and, in particular, the response of the EBL to different time scales of the unsteady geostrophic forcing have been less studied. Lewis and Belcher (2004) presented the most general and rigorous approach for solving the unsteady EBL mean momentum conservation equations with an unsteady pressure gradient and with height-varying viscosity. This and similar approaches (e.g., Shapiro and Fedorovich 2010; Van de Wiel et al. 2010) can derive solutions for a limited range of conditions and often result in intricate expressions that can be hard to interpret physically. Simpler analytical solutions, if available, would therefore be useful.

In this paper, we conduct a suite of LES studies where a neutral EBL flow is forced by a time-varying mean horizontal pressure gradient. The unsteady pressure gradients that are of interest here would be associated with mesoscale variability at time scales of about $0.5-24 \mathrm{~h}$ and horizontal scales of about $5-200 \mathrm{~km}$, resulting, for example, from moving fronts, convective systems, and jet stream excitations (Nastrom and Fritts 1992; Fritts and Nastrom 1992). Various idealized regimes for forcing variability are investigated, focusing on the effect of the variability time scale relative to the characteristic time scale of the mean EBL flow. We then propose a simple physical model for the response of the EBL in unsteady conditions. The reduced model is analytically solvable for a wide range of conditions and is here validated against the LES results. The model is derived analytically from the Reynolds-averaged Navier-Stokes (RANS) equations of motion and results in a second-order ordinary differential equation (ODE) that suggests that the dynamics of the unsteady EBL are analogous to those of a damped oscillator. We specifically use a mass-spring-damper (MSD) system, as the most common and familiar example of a damped oscillator, to further discuss this analogy.

\section{Large-eddy simulations}

\section{a. Physical and numerical details of the code}

The numerical simulations rely on the LES technique, which resolves the large scales of motion and parameterizes eddies smaller than the grid-filter size. The rationale for the approach is that the large eddies contain most of the energy of the flow [ideally over $80 \%$ for LES (Pope 2000)] and account for most of the turbulent fluxes. The LES code used here solves the incompressible continuity equation (in fact, an equivalent Poisson equation for pressure) and the Navier-Stokes momentum equations in a neutral EBL, given, respectively, by

$$
\begin{gathered}
\frac{\partial \tilde{u}_{i}}{\partial x_{i}}=0 \\
\frac{\partial \tilde{u}_{i}}{\partial t}+\tilde{u}_{j}\left(\frac{\partial \tilde{u}_{i}}{\partial x_{j}}-\frac{\partial \tilde{u}_{j}}{\partial x_{i}}\right)= \\
-\frac{1}{\rho} \frac{\partial \tilde{p}^{*}}{\partial x_{i}}-\frac{\partial \tau_{i j}}{\partial x_{j}}+f_{c}\left(U_{g}-\tilde{u}_{1}\right) \delta_{i 2} \\
-f_{c}\left(V_{g}-\tilde{u}_{2}\right) \delta_{i 1}
\end{gathered}
$$

where $x_{i}$ is the position vector; $\tilde{u}_{i}=(\tilde{u}, \tilde{v}, \tilde{w})=\left(\tilde{u}_{1}, \tilde{u}_{2}, \tilde{u}_{3}\right)$ is the resolved velocity vector; $\tau_{i j}$ is the deviatoric part of the subgrid-scale (SGS) stress tensor; $f_{c}$ is the Coriolis parameter, taken here as $1.394 \times 10^{-4} \mathrm{~Hz}$ (this corresponds to latitude of $73^{\circ}$, but its absolute value is not particularly relevant, as we argue later); and $\delta_{i j}$ is the Kronecker delta. The mean horizontal pressure forcing that we vary in these simulations is represented in Eq. (2) as an equivalent geostrophic wind vector $\left(U_{g}, V_{g}\right)$. A pseudospectral approach in the horizontal directions and a second-order centered-difference scheme in the vertical direction are used for the numerical evaluation of the spatial derivatives. For time advancement, the fully explicit second-order Adams-Bashforth method is applied. Because of the assumption of neutral static stability, no buoyancy forces are included, and thus no energy conservation equation is needed for the present paper. The subgrid scales' influence on the larger resolved scales, given by the divergence of $\tau_{i j}$, is parameterized using the scale-dependent Lagrangian dynamic subgrid-scale model (Bou-Zeid et al. 2005). The full details of the code, along with extensive validation for neutral flows with the same SGS model can be found in Bou-Zeid et al. (2005).

The total vertical Reynolds stresses $R_{i 3}(i=1,2$ only) in LESs are given by

$$
R_{i 3}=-\left(\overline{u_{i} u_{3}}-\overline{u_{i}} \overline{u_{3}}\right)-\tau_{i 3}
$$

and consist of a resolved component (first term in the equation) and a subgrid-scale one (second term in the 
TABLE 1. Details of the five LES runs. Time $t$ is in hours.

\begin{tabular}{ll}
\hline \multicolumn{1}{c}{ Case } & \multicolumn{1}{c}{ Forcing variability } \\
\hline 1. Very fast oscillation & $U_{g}=\cos (2 \pi t / 1), V_{g}=\sin (2 \pi t / 1)$ \\
2. Very slow oscillation & $U_{g}=\cos (2 \pi t / 25), V_{g}=\sin (2 \pi t / 25)$ \\
3. Resonant frequency & $U_{g}=\cos (2 \pi t / 12.5), V_{g}=0$ \\
4. Step change & $U_{g}(t \leq 25)=1, U_{g}(t>25)=2, V_{g}=0$ \\
5. Linear change & $U_{g}(t)=t / 50+1, V_{g}=0$ \\
\hline
\end{tabular}

equation). The overbar denotes Reynolds averaging. These total stresses, which are not found to be constant with height even in the surface layer of the simulations (the constant stress layer being, at best, a crude assumption), are used later to compute the vertical eddy viscosity:

$$
\nu_{T}=\frac{\sqrt{R_{13}^{2}+R_{23}^{2}}}{\sqrt{(d u / d z)^{2}+(d v / d z)^{2}}} .
$$

\section{b. The suite of simulations}

We performed five simulations of unsteady EBLs. Simulations 1-3 are used in this section to examine the dependence of the EBL response on the frequency of the imposed forcing, relative to the natural frequency of the EBL, and to elucidate the dynamics of unsteady EBL flows. Simulations 3-5 are used in the following section to validate our analytical model for the neutral unsteady EBL (see Table 1). The height of the domain in all simulations is $L_{z}=2000 \mathrm{~m}$, and its horizontal extent is $2 \pi L_{z} \times 2 \pi L_{z}$. The simulations are carried out for $50 \mathrm{~h}$ (physical time), including warm-up, on a $128 \times 128 \times$ 128 gridcell numerical mesh. The lateral boundary conditions are periodic, as dictated by the use of pseudospectral numerical methods; a stress-free and impermeable $(w=0)$ upper boundary condition is used. At the lower boundary (the wall), a logarithmic law wall model is used with a surface roughness length of $0.01 \mathrm{~m}$ (representing an open flat terrain of low roughness). The code solves a set of equations that has been normalized by the geostrophic wind magnitude at $t=0 \mathrm{~h}$ of $8 \mathrm{~m} \mathrm{~s}^{-1}$ and by a typical boundary layer height scale (here selected as $z_{i}=1 \mathrm{~km}$, but its value is irrelevant since no inversion is imposed and the Ekman layer has the full domain height of $2 \mathrm{~km}$ to adjust to the geostrophic conditions). Viscous terms are neglected, implying an infinite Reynolds number.

In Table 1, as in Eq. (2), the spatially homogeneous, time-variable pressure gradient is represented as an equivalent horizontal geostrophic wind with components $U_{g}$ (streamwise) and $V_{g}$ (cross stream):

$$
\left[U_{g}(t), V_{g}(t)\right]=\left[-\frac{1}{f \rho} \frac{\partial p(t)}{\partial y}, \frac{1}{f \rho} \frac{\partial p(t)}{\partial x}\right] .
$$

The initial conditions of all the simulations are set as $u(t=0, z)=U_{g}(0)=G(0)$ and $v(t=0, z)=V_{g}(0)=0$. A warm-up period is included to allow turbulence to develop, but as we will illustrate later, the influence of the initialization is still observed in the statistics of these unsteady flows.

\section{c. LES results}

The pressure forcing for cases 1 and 2, as can be seen in Table 1 , is given by

$$
\begin{gathered}
U_{g}(t)=G(0) \cos \left(2 \pi t / \tau_{f}\right), \\
V_{g}(t)=G(0) \sin \left(2 \pi t / \tau_{f}\right),
\end{gathered}
$$

where $t$ is time, $\tau_{f}$ is the period of the variable pressure forcing ( $1 \mathrm{~h}$ for case 1 and $25 \mathrm{~h}$ for case 2 ), and $G(0)=$ $G=1$ is the magnitude of the nondimensional geostrophic wind vector. In both cases 1 and 2, the magnitude of the pressure forcing is constant, but its direction is rotating with a period equal to $\tau_{f}$. Note that all results in the paper, as in the LES code, are normalized by the initial value of dimensional geostrophic wind magnitude $G_{d}(0)$, given here a value of $8 \mathrm{~m} \mathrm{~s}^{-1}$. However, since the code assumes an infinite Reynolds number, the dependence of the normalized results on the dimensional magnitude of $G_{d}$ is only through the Rossby number $\left[\mathrm{Ro}=G_{d}(0) / L f_{c}\right]$. The dimensional values used here result in $\mathrm{Ro} \approx 28$, a typical value for atmospheric boundary layers except at mid- to high latitudes (Fleagle and Businger 1981). This Ro will remain fixed in all LES runs since we do not expect the results to be highly sensitive to moderate change in its magnitude (though the analytical model presented later can be solved for any Ro). However, the results will not apply near the equator where $f_{c} \rightarrow 0$, as explained in section $4 \mathrm{~b}$.

In the absence of frictional forces, the natural frequency of inertial oscillations in geophysical flows is usually equal to the Coriolis parameter $f_{c}$, which in our simulation is $1.394 \times 10^{-4} \mathrm{~Hz}$. This frequency characterizes the response time of the mean flow, and the natural time scale of the EBL is thus $\tau_{\mathrm{EBL}}=2 \pi / f_{c} \approx$ $12.5 \mathrm{~h}$ (Tennekes and Lumley 1972). The addition of a dissipative force could alter the natural frequency of such systems; we will come back to this point later in the paper (section $3 b$ ). As such, the choice of $f_{c}$ also influences the inertial oscillation period and would influence the results beyond its role in Ro. However, this influence is not through its absolute value but, in fact, only through its magnitude relative to the forcing frequency $\tau_{f}$ or, equivalently, through the ratio of the corresponding time periods. In simulation 1 , the time period of the forcing is $\tau_{f}=1 \mathrm{~h}<\tau_{\mathrm{EBL}}$. The $u$ time series produced by the LES (Fig. 1, top-left panel) clearly 

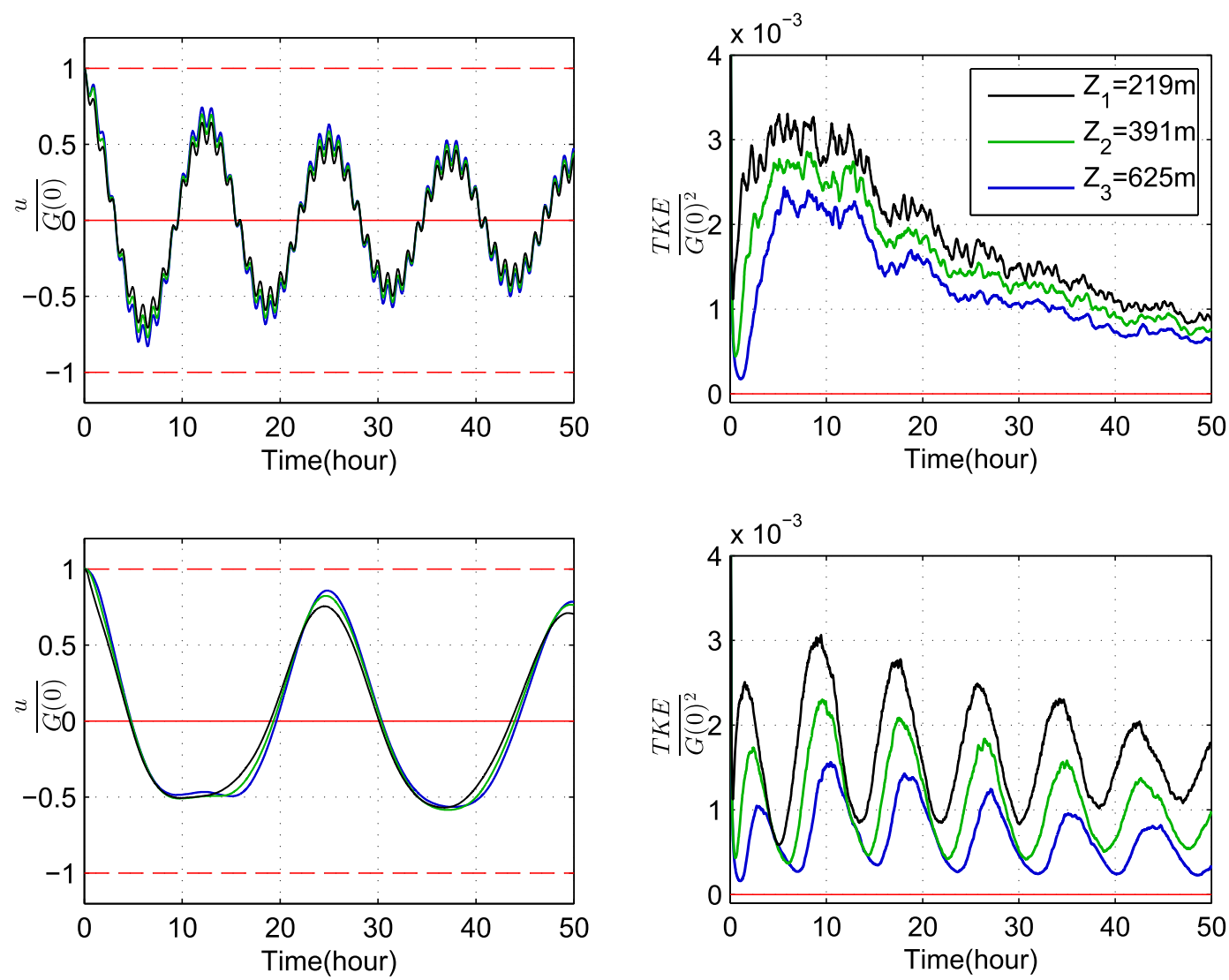

FIG. 1. (left) Time series of mean streamwise velocity, normalized by the geostrophic wind at $t=0 \mathrm{~h}, G(0)$, for (top) fast and (bottom) slow variable forcing. (right) Time series of normalized TKE, normalized by $G^{2}(0)$, for (top) fast and (bottom) slow variable forcing. Three sample heights $Z_{1}, Z_{2}$, and $Z_{3}$ are shown.

illustrates that the EBL cannot respond completely to the cycles of this fast imposed sinusoidal forcing. One can further observe that the EBL displays a series of small oscillations, corresponding to the forcing frequency, superimposed on large oscillations that are related to the EBL natural time scale and that emanate from the initial conditions not matching the equilibrium state of the flow. On the other hand, the turbulent kinetic energy [i.e., TKE $=1 / 2\left(\overline{u_{i} u_{i}}-\overline{u_{i}} \overline{u_{i}}\right)$ with summation over the repeated indices] responds faster than the mean flow to these oscillations (Fig. 1, top-right panel) since the turbulence time scale $\left(\tau_{\text {turb }} \approx z_{i} / u_{*} \sim 1 \mathrm{~h}\right.$, where $u_{*} \sim 0.25 \mathrm{~m} \mathrm{~s}^{-1}$ is the friction velocity and $z_{i} \sim$ $1000 \mathrm{~m}$ is the approximate depth of the EBL) is much shorter than the natural time scale of mean EBL winds. We compute the TKE by averaging in space and time. To obtain the TKE time series, first a spatial average over the horizontal directions is applied to acquire a single velocity profile, and then profiles are averaged in time over a short period of $50 \mathrm{~s}$. A similar procedure is applied to produce velocity time series. Note the small variations in the TKE at the frequency of the forcing, while the large-scale trends are due to initialization and warm-up. This also suggests that the turbulence might be close to quasi equilibrium, even in such flows where the mean is changing relatively rapidly.

In the second case, the time period of the forcing is $\tau_{f}=25 \mathrm{~h}>\tau_{\mathrm{EBL}}$. Therefore, the EBL has enough time to adapt itself to the applied variations. The corresponding $u$ time series in the bottom-left panel of Fig. 1 show that the EBL responds fully to any sinusoidal cycle of the forcing. A single oscillation, with the frequency of the imposed forcing, is observed in the velocity time series. The TKE also responds very fast to the slow forcing, and its peaks and troughs correspond exactly to the peaks and troughs of the mean wind speed $M=\left(u^{2}+v^{2}\right)^{1 / 2}$ (not shown here). Also note that, from this point forward, the tildes and overbars are omitted for simplicity from the symbols of the mean and filtered simulated velocities.

Case 3 is one where we impose a harmonic forcing with $\tau_{f}=12.5 \mathrm{~h} \approx \tau_{\mathrm{EBL}}$ to investigate the modes and dynamics that can potentially be excited by "resonance." Resonance would manifest itself as large-amplitude 

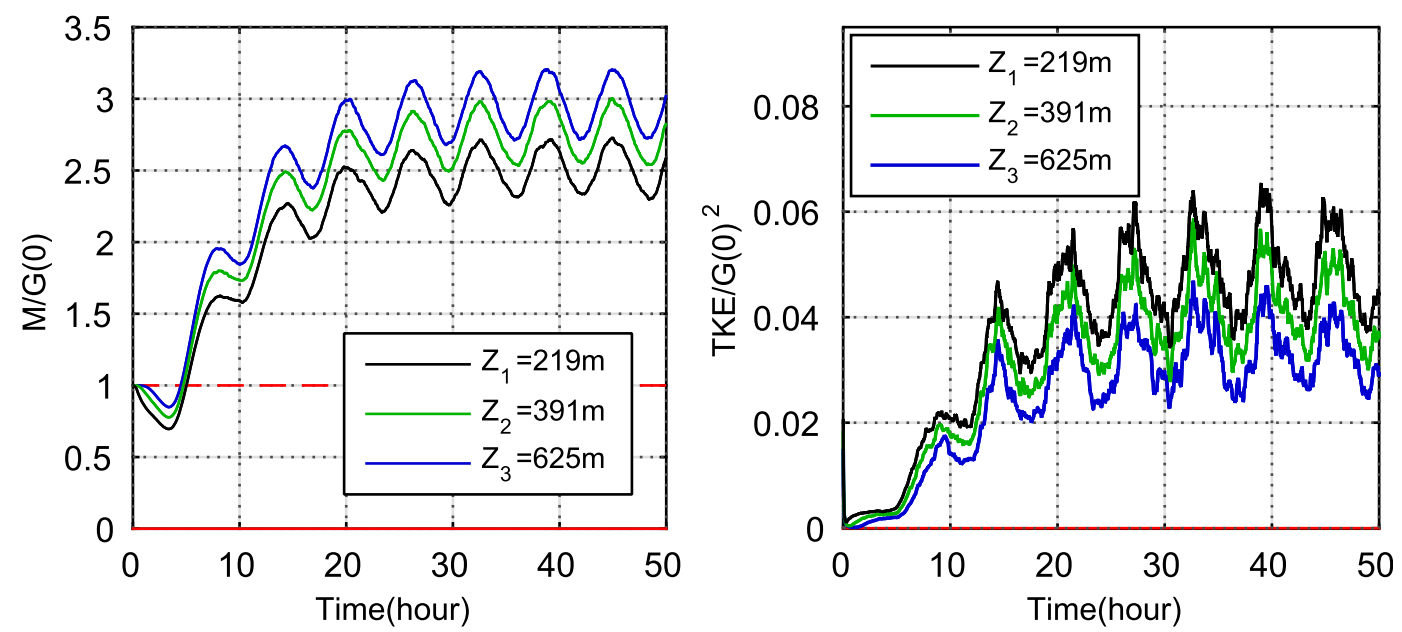

FIG. 2. Normalized mean wind velocity and TKE time series for a resonant forcing (case 3).

oscillations producing velocities in the EBL that exceed the geostrophic wind magnitude. Unlike cases 1 and 2, the magnitude of the pressure forcing here is variable, but its direction is constant in time. Even though the imposed pressure force is sinusoidal and corresponds to a normalized geostrophic velocity magnitude that cannot exceed 1 , Fig. 2 depicts supergeostrophic velocity magnitudes in the EBL that significantly exceed $G=1$. While this appears qualitatively similar to the supergeostrophic winds in the Blackadar and associated analyses, it is produced by very different conditions. Instead of a sudden drop in friction as in the Blackadar solution, here the supergeostrophic winds are the result of resonance (system transmissibility greater than 1) in the response of the EBL when the forcing time scale matches its natural response time scale $\left(\tau_{f} \approx \tau_{\mathrm{EBL}} \approx\right.$ $2 \pi / f_{c}$ ), with no change in stability. The TKE time series in the bottom-right panel of Fig. 2 shows an increasing trend, indicating a rise in the system energy that is also typical of resonance; however, for later times, the TKE does not continue to grow.

These results indicate that the EBL response is highly dependent on the forcing variability regime, and each imposed forcing frequency generates a distinct $u-v$ pattern (see appendix A for the flow hodographs). These time series for cases $1-3$, and particularly for case 3 , are strongly reminiscent of the behavior of an underdamped MSD system. This is not entirely surprising since the Blackadar solution where the friction force is completely removed is equivalent to a mass-spring system (with no damper) and since a recent investigation by $\mathrm{Du}$ and Rotunno (2014) that further elaborates on the Blackadar mechanism for low-level jet formation also hints at damped oscillations in the nocturnal EBL. In the following sections, we aim to formalize this EBL-MSD analogy, to exploit it to develop a model of "maximum simplicity" for the unsteady EBL, and to validate this model using LES.

\section{Reduced model of the EBL}

\section{a. The phenomenology of the EBL-MSD analogy}

If one considers a column of air extending from the surface to some level $z$ (or an oceanic column of water) as a large mass, exerting a (pressure) force on this mass will cause it to start moving; the motion will then generate a Coriolis force and a friction force that will resist this motion. The mean kinetic energy generated by the forcing will be dissipated or damped only by the friction force through surface stress at the ground and through conversion from mean kinetic energy (MKE) to TKE. The Coriolis force is conservative (just like a spring force) and will not destroy MKE (Stull 1988); it can only transfer MKE between the different velocity components $(u$ and $v)$. That is, if the forcing of the column of air produces an increase in the MKE associated with $u$, then Coriolis can only transfer that energy to the $v$ component, where it is stored as kinetic energy in $v$ that appears as potential energy to $u$. Thus, each component acts as a reservoir of potential energy for the other, and conversion between MKE and potential energy for a given component is through the action of the Coriolis force. This effect can be perceived as the spring impact in an MSD system. Hence, one can hypothesize that the unsteady EBL behaves similarly to an MSD system (or, more precisely, as two coupled MSD systems for $u$ and $v$ ), the governing equation of which is

$$
F(t)=m \ddot{x}+B \dot{x}+k x,
$$

where $F(t)$ is the variable forcing; $x$ is the displacement of the mass from the equilibrium position; each dot 
above denotes differentiation once with respect to time; $m$ is the mass; $B$ is the damping coefficient; and $k$ is the spring constant. The solution of this ODE and the resulting MSD dynamics have three regimes based on the value of the damping ratio $\zeta=B /(4 m k)^{1 / 2}$ : an underdamped case $(\zeta<1)$, an overdamped case $(\zeta>1)$, and a critical damping regime $(\zeta=1)$. In the underdamped case (which seems to apply to the EBL, since it is the only regime where resonance occurs for an MSD), the MSD system will oscillate at the natural damped frequency $\omega_{d}=\omega_{n} \sqrt{1-\zeta^{2}}$, which depends on $\zeta$ and on the natural frequency of the undamped system $\omega_{n}=\sqrt{\mathrm{k} / \mathrm{m}}$. If a periodic force that is exerted has a frequency $\omega_{f} \approx \omega_{d}$, resonance will occur. The homogenous solution of the initial value problem given by Eq. (7) in an underdamped, unforced [i.e., with $F(t)=0$ ] case is

$$
x_{h}(t)=e^{-(B / 2 m) t}\left[A \cos \left(\omega_{d} t\right)+B \sin \left(\omega_{d} t\right)\right],
$$

where $A$ and $B$ are determined from the initial conditions.

Critical damping occurs when the system converges to equilibrium (zero displacement for an MSD) as fast as possible without any oscillations. In the overdamped case, the damping slows the convergence of the system from its initial position to its equilibrium position, and the system takes a longer time to converge but does not oscillate. Both of these cases do not display any resonant behavior. Their general solutions, given respectively for the critically damped and overdamped case, are as follows:

$$
\begin{aligned}
& x_{h}(t)=e^{-\omega_{n} t}(A+B t) \quad \text { and } \\
& x_{h}(t)=A e^{c_{1} t}+B e^{c_{2} t},
\end{aligned}
$$

where $C_{1}$ and $C_{2}$ are the two real roots of the associated characteristic equation, and $A$ and $B$ are determined by the initial conditions. While the response of the neutral EBL in our LES appears to correspond to an underdamped system, unstable conditions can increase the turbulent stress term, which is analogous to the damping term, and potentially drive the system to the overdamped regime, although we do not investigate this possibility in this paper.

\section{b. Derivation of a reduced model from the Reynolds-averaged Navier-Stokes equations}

To formalize the EBL-MSD analogy, we start with the RANS equations (since we are only interested in the mean flow) for a planar homogeneous EBL flow:

$$
\begin{aligned}
& \frac{\partial u}{\partial t}=-f_{c}\left(V_{g}-v\right)+\frac{\partial}{\partial z}\left(\overline{u^{\prime} w^{\prime}}\right), \\
& \frac{\partial v}{\partial t}=f_{c}\left(U_{g}-u\right)+\frac{\partial}{\partial z}\left(\overline{v^{\prime} w^{\prime}}\right),
\end{aligned}
$$

where $u$ and $v$ are the mean horizontal velocity components at height $z$ and time $t$ (for a laminar flow, one simply needs to replace the Reynolds stress divergence with its molecular counterpart). The nonlinear advective terms are omitted because of the horizontal homogeneity and small subsidence $w$.

Given the fast response of turbulence depicted in section 2 , one can expect it to remain in quasi equilibrium with the time-varying mean flow (i.e., the turbulence statistics at any point in time are equivalent to those that would be generated if the current mean forcing at that same instant were steady). If such is the case, then turbulence statistics are expected to scale with the mean variables (since both depend in a fixed way on the same characteristic velocity and length scales). For instance, the turbulent stress terms could be modeled using the mean velocities as

$\frac{\partial}{\partial z}\left(\overline{u^{\prime} w^{\prime}}\right) \simeq \frac{\partial}{\partial z}\left(\nu_{T} \frac{\partial u}{\partial z}\right)=\frac{\partial \nu_{T}}{\partial z} \frac{\partial u}{\partial z}+\nu_{T} \frac{\partial^{2} u}{\partial z^{2}} \simeq-\alpha(z, t) u(z, t)$

and

$\frac{\partial}{\partial z}\left(\overline{v^{\prime} w^{\prime}}\right) \simeq \frac{\partial}{\partial z}\left(\nu_{T} \frac{\partial v}{\partial z}\right)=\frac{\partial \nu_{T}}{\partial z} \frac{\partial v}{\partial z}+\nu_{T} \frac{\partial^{2} v}{\partial z^{2}} \simeq-\alpha(z, t) v(z, t)$,

where $\nu_{T}$ is an eddy viscosity and $\alpha$ is an inverse time scale. The exact expressions of both $\nu_{T}$ and $\alpha$ and their relation to each other depend on the similarity theory that best describes the dynamics of the flow (e.g., log law, defect law, or Rossby number similarity). This parameterization of the stress terms is, in fact, a very classic model of their divergence as being proportional to the velocity at the same height (e.g., Schmidt 1947; Haurwitz 1947). For example, Schmidt (1947) notes that frictional force per unit mass could be written as velocity times an expression that is a function of height. Baker et al. (1987), Polavarapu (1995), and $\mathrm{Pu}$ and Dickinson (2014) have also used similar models for the friction forces in Ekman flows. Note that $\alpha(z, t)$ in its general form is both a function of height and time. But here we postulate that its time-averaged value $\bar{\alpha}(z)$ is sufficient for the current model (an assumption to be validated later), and we will use it herein [omitting the overbar and writing it as $\alpha(z)$ ]. The equations with a time-varying $\alpha$ were solved as well, but we leave this for a future investigation since it is not critical for the purpose of this paper, where we intend to exhibit the simplest model for unsteady Ekman flows.

If turbulence is not in perfect equilibrium with the mean flow, Eq. (11) could be still tested as a model for mathematical convenience, but it would result in errors that need to be quantified for a given flow. Equation (11) is a general relation, but more specific forms can be derived: 
for example, for the log law (or other similarity laws), as follows. The gradient of a velocity component (e.g., $u$ ) in the lower part (surface layer) of a neutral atmospheric EBL (which best characterizes our LES) can be expressed using the logarithmic wind profile (Pope 2000):

$$
\frac{\partial u}{\partial z}=\frac{u_{*, x}}{\kappa z} ; \quad u(z)=\frac{u_{*, x}}{\kappa} \ln \left(\frac{z}{z_{0}}\right),
$$

where $\kappa$ is the von Kármán constant, $z_{0}$ is the surface roughness, and $u_{*, x}$ is the component of the friction velocity associated with the surface stress in the $x$ direction only. Using the expression of the gradient of Eq. (12) and replacing $u_{*, x}$ from the $\log$ wind profile also given by Eq. (12) into the turbulent stress divergence terms in Eq. (10) yields

$$
\frac{\partial}{\partial z}\left(\nu_{T} \frac{\partial u}{\partial z}\right)=\frac{u_{*, x}}{\kappa} \frac{\partial}{\partial z}\left(\frac{\nu_{T}}{z}\right)=\frac{u(z)}{\ln \left(z / z_{0}\right)} \frac{\partial}{\partial z}\left(\frac{\nu_{T}}{z}\right) .
$$

The dependence on $z$ in the numerator and denominator of $u(z) / \ln \left(z / z_{0}\right)$ cancels out. The exact same derivation can be made for the other velocity component $v$, giving a relation similar to Eq. (13). We may then define $\alpha(z)$ as

$$
\alpha(z) \equiv-\frac{1}{\ln \left(z / z_{0}\right)} \frac{\partial}{\partial z}\left[\frac{\nu_{T}(z)}{z}\right] .
$$

Either $\nu_{T}$ or $\alpha$ can be parameterized based on established similarity theories and/or LES [one can be computed from the other via Eq. (14) or comparable relations for other similarity laws]; the parameterized variable becomes the only parameter that needs to be calibrated or imposed in the proposed model. Equation (14) is a special case of Eq. (11) but has the advantage of providing an explicit and direct relation between $\nu_{T}$ and $\alpha$. While the use of the log law restricts this derivation to the lower $15 \%$ or so of the EBL, an alternative derivation using a defect law for the higher elevations in fact gives a comparable result (the turbulent stress terms remain proportional to the velocities at the same height) but with a factor $\alpha(z)$ slightly different from the one in Eq. (14). Thus, the closure given in Eq. (11) is valid throughout the EBL, with appropriate $\alpha$ for different layers or different conditions, provided a given similarity theory can be used to relate the turbulence to the mean.

Substituting Eq. (11) into Eq. (10) and differentiating once with respect to time, we obtain

$$
\begin{aligned}
& \frac{d^{2} u}{d t^{2}}+\alpha \frac{d u}{d t}+f_{c}^{2} u+\alpha f_{c} v=f_{c}^{2} U_{g}-f_{c} \frac{d V_{g}}{d t} \quad \text { and } \\
& \frac{d^{2} v}{d t^{2}}+\alpha \frac{d v}{d t}+f_{c}^{2} v-\alpha f_{c} u=f_{c}^{2} V_{g}+f_{c} \frac{d U_{g}}{d t}
\end{aligned}
$$

This resulting reduced form of the RANS equations confirms that each horizontal velocity component obeys an equation exactly similar to an MSD system [cf. with Eq. (7)]. Note that $\alpha$, which arises from frictional forces, corresponds to the damper coefficient, while $f_{c}$, which is associated with the Coriolis force, corresponds to the spring coefficient, as postulated before. However, these two velocities or MSD systems are coupled, and this will give rise to important special characteristics. We note that the additional differentiation in Eq. (15) is not required to obtain a mathematical solution, but we apply it here to reveal the analogy to the mass-spring-damper system. We now define the complex variable $A \equiv u+i v$, as often done for the Ekman equations, to combine Eqs. (15a) and (15b) into

$$
\frac{d^{2} A}{d t^{2}}+\alpha \frac{d A}{d t}+f_{c}\left(f_{c}-i \alpha\right) A=f_{c}^{2} A_{g}+i f_{c} \frac{d A_{g}}{d t} .
$$

The fact that Eqs. (15) and (16) are ODEs can be traced to the separation of the dependence on $z$ and $t$ that the model in Eq. (11) allows: the gradients of $u$ and $v$ (i.e., the dependence of $u$ and $v$ on $z$ ) are now an imposed closure that is fully determined by the velocities at that height and time, albeit a very flexible one that can vary for different layers of the EBL [through the variation of $\alpha(z)]$. The ODE can hence be solved at each $z$ independently, and $z$ becomes a parameter rather than an independent variable. The homogeneous solution of this complex ODE is

$$
A_{h}(t)=C_{0} e^{-\alpha t}\left(\cos f_{c} t-i \sin f_{c} t\right),
$$

where $C_{0}$ is determined from initial conditions. The full general solution of Eq. (16) also requires particular solutions associated with the forcing term that we will derive later. The complete mathematical homogenous solution of Eq. (16) should have an additional undamped harmonic term; however, it can be eliminated (its coefficient will be zero) because of the nature of the physical system (in order for the MSD to forget the initial conditions as $t \rightarrow \infty$ ). A mathematical proof that the undamped harmonic oscillator coefficient has to always be zero is exhibited in appendix B. The coupling of the two velocities results in some characteristics of the solution that are different from those of simple damped oscillators, such as an MSD system: the coefficient $f_{c}$, which is the natural frequency of the undamped system, also emerges as the natural frequency of this coupled damped system, which thus does not depend on the damping coefficient as for a single MSD. This point suggests that inertial oscillations in the EBL always occur at the Coriolis frequency $f_{c}$ of that latitude. At high latitudes, the Coriolis frequency increases, and thus the 
natural inertial time scale of the EBL decreases; whereas, at lower latitudes, the EBL time scale of the oscillations increases since the Coriolis effect reduces to about zero near the equator where the velocity coupling is at its minimum value. Changes in $f_{c}$ with latitude can change the Rossby number Ro (but not drastically once the geostrophic wind and the EBL height are set, except near the equator) as well as $\tau_{\mathrm{EBL}} / \tau_{f}$. We change the time scale (or frequency) ratio here by changing $\tau_{f}$ rather than $\tau_{\mathrm{EBL}}$. While the changes in Ro are not explicitly considered (not expected to be significant away from the equator), the analytical model can be solved for any Ro.

We should also underline here that, although dampedoscillator models exist for other geophysical flows (e.g., for parcel oscillation in a stably stratified medium with viscosity), the physical forces involved are very different in our unsteady EBL case. Moreover, compared to related previous treatments of the unsteady EBL, the main distinguishing features of our model are as follows:

1) Our derived equations allow us to vary the forcing as well as the turbulent viscosity with height and time, while previous studies (e.g., Pollard and Millard 1970) have represented the EBL as a vertically integrated slab layer. Therefore, our model can be applied not only in barotropic EBLs, but also in baroclinic cases where pressure gradients are changing with height (with appropriate expressions of $\alpha$ for such flows) since the proposed model treats each height or layer of the EBL as an independent MSD controlled by its unique damper and spring (and also since baroclinicity typically occurs at synoptic horizontal spatial scales and does not violate our small- to mesoscale horizontal homogeneity assumption).

2) The governing equation [Eq. (16)] can be solved for a very wide range of forcing regimes associated with $A_{g}(t)$, as long as $A_{g}(t)$ is piecewise differentiable and particular solutions for Eq. (16) can be found. The governing equation can also be solved regardless of how the eddy viscosity varies with height as long as the vertical derivative $\partial\left[\nu_{T}(z) z^{-1}\right] / \partial z$ appearing in Eq. (14) can be computed or approximated at the given height. Even time variation of the eddy viscosity can be accounted for (the governing equation then simply becomes an ODE with nonconstant coefficients). Thus, the simplicity of the model allows it to be analytically solvable over a wider range of conditions than the more exact direct solutions of the two coupled partial differential equations [Eq. (10), as done in Lewis and Belcher (2004)].

3) The formal linking of the $\alpha$ parameter to eddy viscosity [Eq. (14) for the log law or corresponding relations for other conditions], the investigation of the physical analogy with an MSD, and, very importantly, the model validation against LES that we conduct later in this paper provide a generalizable and reliable framework that will be useful for other researchers developing or using related models of unsteady flows in the atmosphere (e.g., Pollard and Millard 1970; Du and Rotunno 2014).

\section{c. Validation of an optimized MSD model against LES}

The particular solutions to this problem depend on the type of the forcing we consider. Three cases (cases 3-5 in Table 1) have been derived here, and the solutions obtained are used to evaluate the model by comparing to LES results. In validating the model, as a first step we aim to confirm that the simplifications that result in the model structure [the ODE in Eq. (16)] are applicable. To that end, we simply compute for each height, in each case, the coefficient $\alpha(z)$ that minimizes the root-meansquare error (RMSE) for a given model output with respect to LES results (further details are provided later in this paper). We then compute the solution with that optimized coefficient, and the results are herein referred to as the optimized MSD (OMSD).

The general form of the sinusoidal forcing $A_{g}$ in case 3 and its particular solution $A_{p}$ are given by

$$
\begin{aligned}
A_{g} & =Q \cos (\omega t) \\
A_{p}(t) & =\frac{Q f(f-i \alpha)}{(f-i \alpha)^{2}-\omega^{2}} \cos (\omega t)-\frac{i Q \omega f}{(f-i \alpha)^{2}-\omega^{2}} \sin (\omega t) .
\end{aligned}
$$

Case 3, as we noted before, displays resonance and as such is a challenging case for the model. The LES and OMSD results for that case can be compared in Fig. 3 (top panel). The optimization here, and in the rest of this section, minimizes the RMSE of $u$ since it is the component we are validating. As can be seen, the match between the LES and OMSD at multiple heights is remarkably good given how simplified the OMSD equations are compared to the nonlinear PDEs solved by the LES.

The step-change forcing and its particular solution could be found as follows:

$$
\begin{aligned}
& A_{g}= \begin{cases}a, & t \leq t_{c} \\
b, & t>t_{c}\end{cases} \\
& A_{p}=u_{p}+i v_{p}=\left\{\begin{array}{ll}
\frac{f^{2} a}{f^{2}+\alpha^{2}}+i \frac{\alpha f a}{f^{2}+\alpha^{2}}, & t \leq t_{c} \\
\frac{f^{2} b}{f^{2}+\alpha^{2}}+i \frac{\alpha f b}{f^{2}+\alpha^{2}}, & t>t_{c}
\end{array} .\right.
\end{aligned}
$$



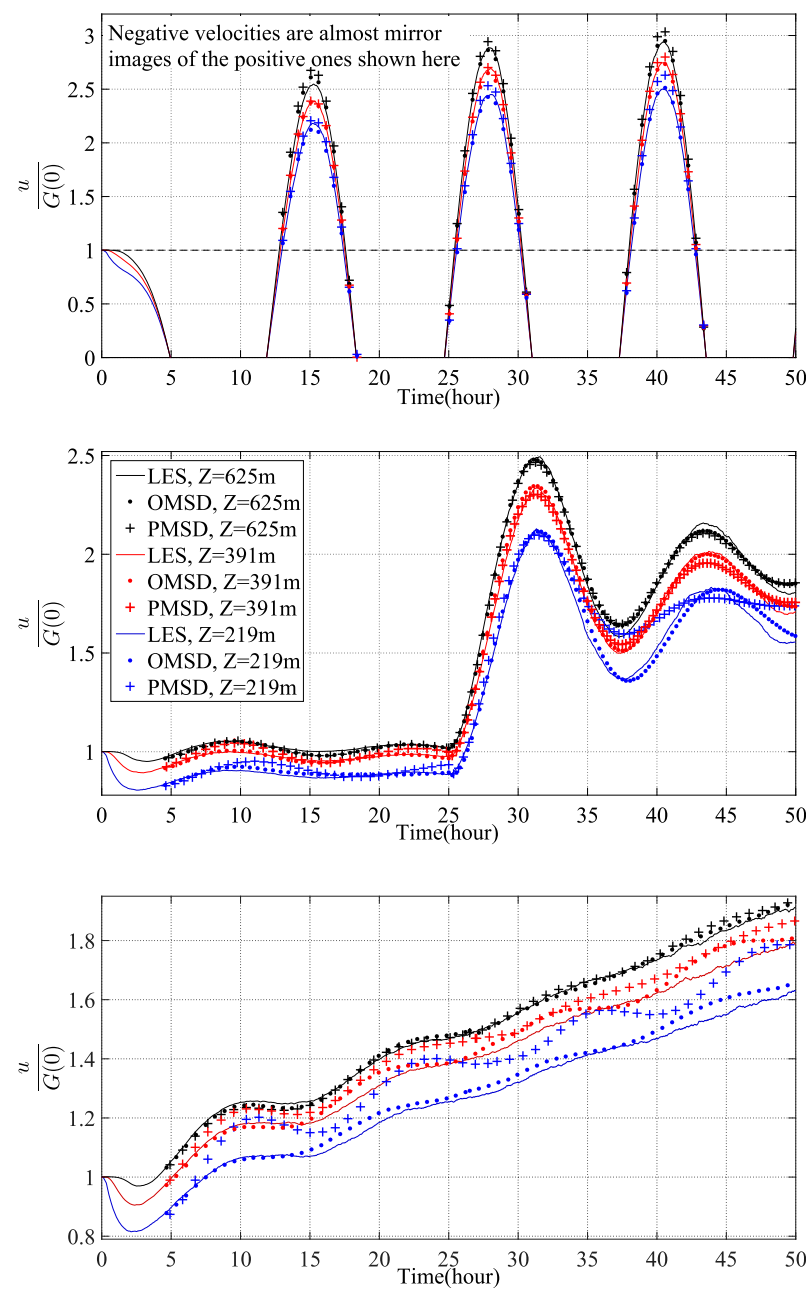

FIG. 3. LES, OMSD, and PMSD velocity for (top) sinusoidal, (middle) step, and (bottom) linear changes in the forcing.

The middle panel of Fig. 3 shows the specific example corresponding to the LES of case 4 , where $t_{c}=25$, $a=1$, and $b=2$. Comparing LES and OMSD here also proves that the model is capable of capturing the LES dynamics quite well for an abrupt jump in the forcing.

The last case studied here is the linear change in the forcing (LES case 5), given with its particular solution by

$$
\begin{aligned}
A_{g} & =(m t+n)+i\left(m^{\prime} t+n^{\prime}\right), \\
u_{p}(t) & =f\left[\left(-m^{\prime} \alpha+m f\right) t-n^{\prime} \alpha-m^{\prime}+n f\right] /\left(f^{2}+\alpha^{2}\right), \quad \text { and } \\
v_{p}(t) & =f\left[\left(m \alpha+m^{\prime} f\right) t+n \alpha+m-n^{\prime} f\right] /\left(f^{2}+\alpha^{2}\right) .
\end{aligned}
$$

LES results for this linear change in the forcing from 1 to 2 over a period of $50 \mathrm{~h}$ are depicted in Fig. 3 (bottom) with $m=1 / 50, n=1, m^{\prime}=0$, and $n^{\prime}=0$. The OMSD captures the LES results well for this slow change in the forcing; however, the match is somewhat inferior to previous cases. This is attributed to the continuous change in the forcing and the use of an averaged $\alpha$ for this case, which does not allow the optimized eddy viscosity to vary with time as it physically should. The equation with a linearly increasing $\alpha$ was also solved analytically as mentioned before, but this is not detailed here since we intend to focus on and test the simplest model for the unsteady EBL to understand its dynamics. Applications of the model where the accuracy of quantitative results is important can and should seek more accurate expressions for $\alpha(t)$, as we will further elaborate later.

\section{d. Validation of a parameterized MSD model against LES}

Using LES results, a turbulent viscosity model for $\nu_{T}$ is developed for our analytical solutions. It can then be used without the optimized $\alpha$ or any LES to apply the MSD model. The resulting model will be referred to as the parameterized MSD (PMSD). The eddy viscosity can be expressed as a mixing eddy length scale $\lambda_{m}(z)$ times the corresponding characteristic velocity scale $U_{m}$ (selected here near $\left|A_{g}\right|$ averaged over some previous time period to capture the turbulence memory). The LES results, from which one can directly compute $\nu_{T}$ via Eq. (4), suggest that the length scale depends parabolically on $z / z_{i}$, with a maximum near the middle of the EBL; $z_{i}$ is the height of the atmospheric EBL imposed in the LES, defined here as $1000 \mathrm{~m}$ and equal to half the domain height. Taking the product of these velocity and length scales and lumping the proportionality coefficients into a characteristic length scale $L_{0}$ yields

$$
\nu_{T}(z)=\overline{\left|A_{g}\right|} L_{0}\left[2 \frac{z}{z_{i}}-\left(\frac{z}{z_{i}}\right)^{2}\right] .
$$

Similar parabolic eddy-viscosity profiles have been previously used by Sitaraman and Shirvaikar (1974), Lentz (2001), and Herrmann and Madsen (2007). The turbulent viscosity was directly calculated from the total Reynolds stresses and velocity gradients produced by the LES [Eq. (4)], which then allows us to calibrate $L_{0}$ for our model [Eq. (21)]. This LEScalibrated $L_{0}$ varied in the range $40-70 \mathrm{~m}$ (somewhat dependent on the forcing and the averaging of $A_{g}$ ), but a representative value of $50 \mathrm{~m}$ is used for all cases here to test the applicability of a universal formulation with a fixed $L_{0}$. Figure 4 depicts the good agreement of this viscosity model with the LES-computed 

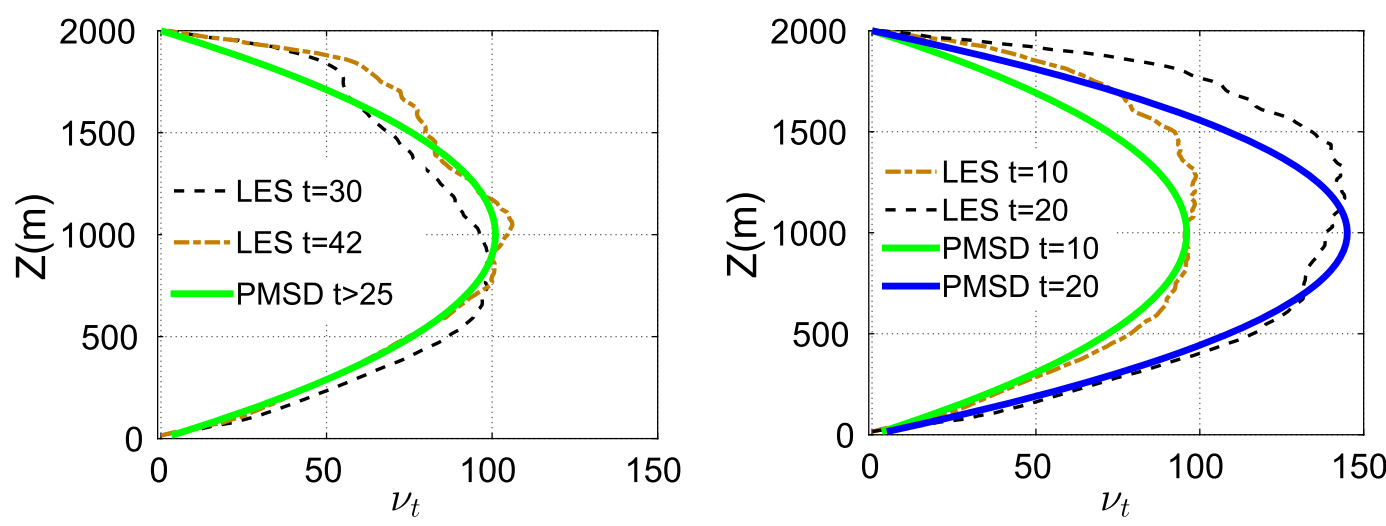

FIG. 4. Proposed eddy-viscosity model for PMSD [Eq. (21)] tested against LESs [Eq. (4)] for the (left) step (case 4) and (right) sinusoidal (case 3) simulations for a fixed $L_{0}=50 \mathrm{~m}$. Each line is for a 1 -h average starting at the time indicated in the legend.

eddy viscosity for two test cases and at two different times.

We can now apply this model, without access to LES, to make forecasts in the EBL, as depicted for cases 3-5 in Fig. 3. The error in the PMSD model is now related both to its structure/assumptions (which was already assessed by the OMSD version) and to the accuracy with which one can obtain $\nu_{\mathrm{T}}$ or $\alpha$ (which did not influence the performance of the OMSD significantly). As with the OMSD, the agreement with LES is better at higher elevations; however, the agreement is generally lesser than for the OMSD. Despite these discrepancies, the proposed reduced model seems to be quite promising in all three investigated cases.

\section{e. Sources of error and their reduction in the MSD model}

While the main aim of this paper is to present and test a general formulation of the model, rather than to fine-tune it for prediction, a brief discussion of the main sources of error and how they can be reduced is in order. Given the better performance of the OMSD, compared to the PMSD, it can be concluded that the accuracy of the parameterized eddy viscosity, or equivalently of $\alpha$, is the main determinant of the accuracy of model predictions (as we will see here, it also influences the skill of the OMSD). However, the parameterization of the turbulent viscosity in the lower atmosphere remains an important and open research challenge. We briefly discuss some potential improvements for the purpose of our model in this section.

First, we compare the full velocity profiles simulated by the LES and modeled by the OMSD \{minimizing the coefficient of variation $(\mathrm{CV})$ of the $u$ component $[\mathrm{CV}(u)]\}$ and the PMSD. This is depicted in the left panel of Fig. 5 at two different times. In addition to the dependence of the error on height, its dependence on time (or forcing phase) is also illustrated by this figure. We selected two times when the error is minimal $(t=$ $42 \mathrm{~h}$, during the acceleration phase) and when it is relatively large ( $t=30 \mathrm{~h}$, near the peak of the velocity). Despite these errors, the figure clearly confirms that the MSD models can capture the bulk features of the velocity profile, as simulated by LES, everywhere in the EBL.

To quantify model error, we will use the CV of the RMSE:

$$
\mathrm{CV}(\psi)=\frac{1}{\bar{\psi}_{\mathrm{LES}}} \sqrt{\frac{\sum_{t=1}^{N}\left[\psi_{\mathrm{MSD}}(t)-\psi_{\mathrm{LES}}(t)\right]^{2}}{N}},
$$

where $\psi$ could be one of the two wind components $u$ or $v$ or the mean wind speed $M$ [recall that, in Fig. 3, the optimization minimized $\mathrm{CV}(u)]$. The percentile $\mathrm{CV}$ $(\mathrm{CV} \times 100)$ profiles, averaged from $t=27$ to $47 \mathrm{~h}$ and calculated based on $u$ and $M$ for case 4 (as an illustrative example), are compared in Fig. 5 (right). While the error resulting from minimization of $\mathrm{CV}(u)$ in the context of the OMSD model remains small and constant at all heights, the one resulting from minimizing $\mathrm{CV}(M)$ increases significantly near the surface. Optimization using $\mathrm{CV}(M)$ attempts to minimize the error in both velocity components simultaneously, but this task becomes more difficult near the surface. This is related to the failure of the Boussinesq analogy, which postulates that the same eddy viscosity applies in the $x$ and $y$ directions and which was made for the PMSD model. But this assumption is problematic near the surface of the EBL primarily because of Ekman turning. Therefore, in order to increase the accuracy of the PMSD model, especially close to the ground, its structure may be 

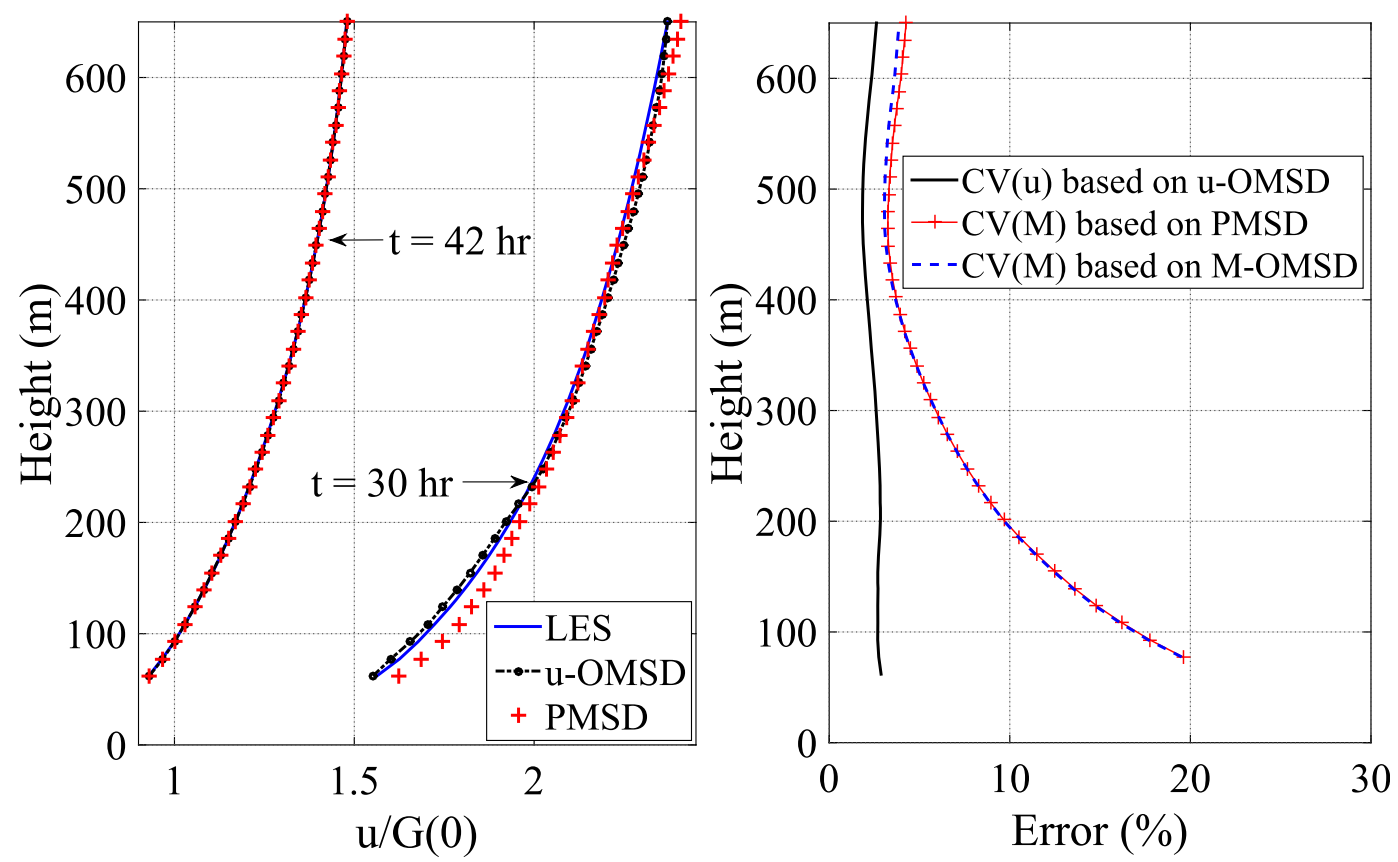

FIG. 5. MSD model accuracy with height for case 4. (left) Model profiles of $u$-OMSD and PMSD vs LES at $t=30$ and $42 \mathrm{~h}$. (right) Comparison of OMSD optimized by minimizing CV $(M)$ ( $M$-OMSD) and hence invoking the Boussinesq analogy (dashed blue line), an OMSD version where $\alpha_{x} \neq \alpha_{y}$ optimized by minimizing CV $(u)(u$-OMSD, as done in the OMSD applications in Fig. 3, solid black line), and CV $(M)$ from the PMSD model using Boussinesq's analogy (solid red line).

altered to allow two different $\nu_{T, x}$ and $\nu_{T, y}$ or $\alpha_{x}$ and $\alpha_{y}$. However, this is outside of the scope of this paper. To further illustrate this dependence of the model on the accuracy of the viscosity model, we compare $\mathrm{CV}(M)$ for the PMSD and the OMSD optimized by minimizing $\mathrm{CV}(M)$ in Fig. 5. The strong similarity between the profiles indicates that the model for turbulent viscosity (in the PMSD) can capture the best possible solution with the current structure of the model, implying that the PMSD performs to the best extent possible in this test given that it was formulated by invoking the Boussinesq analogy.

Another important aspect of the PMSD model is the averaging to compute $\overline{\left|A_{g}\right|}$ needed in Eq. (21). This averaging was over hours $25-50$ for the sudden jump case (case 4), was over the whole period for the linear increase case (case 5), and was taken as the amplitude of the sinusoidal forcing for case 3 . While this might at first seem inconsistent, the choices, in fact, are made to provide the best estimate of the forcing velocity that is responsible for shear turbulence production, and hence for eddy viscosity, during the simulation period of most interest to the PMSD model prediction. More sophisticated approaches for setting the average $A_{g}$ will be investigated in future applications of the model along with a time-varying $\alpha$, but these are of minor importance for demonstrating the validity of the model assumptions and structure.

\section{Further analyses of the unsteady EBL using the reduced model}

\section{a. Lagrangian and energy partitioning of the MSD model}

In the previous section, we derived the ODE governing the response of the EBL and validated it with LES results. The simplicity of the resulting model can be exploited to further explain and illustrate the physics of this unsteady geophysical flow. As an illustration, we consider here an important attribute of these flows: the energy distribution between different components (kinetic vs potential). This distribution can be investigated by deriving the Lagrangian (kinetic minus potential energy) of our system or its Hamiltonian (kinetic plus potential energy). The Hamiltonian, in particular, has been previously used to understand the dynamics of similar atmospheric systems with gravity waves and temperature effects (Hoskins 1975; Durran 1989; Salmon 1983; Dellar and Salmon 2005).

The Lagrangian can be obtained very easily from Eqs. (15a) and (15b) as 


$$
\begin{aligned}
L_{u}[u(z), v(z), \dot{u}(z)] & =\frac{1}{2} \dot{u}(z)^{2}-\frac{1}{2} f^{2} u(z)^{2}-f \alpha(z) u(z) v(z), \quad \phi[\dot{u}(z)]=\frac{1}{2} \alpha(z) \dot{u}(z)^{2} \quad \text { and } \\
L_{v}[v(z), u(z), \dot{v}(z)] & =\frac{1}{2} \dot{v}(z)^{2}-\frac{1}{2} f^{2} v(z)^{2}+f \alpha(z) v(z) u(z), \quad \phi[\dot{v}(z)]=\frac{1}{2} \alpha(z) \dot{v}(z)^{2},
\end{aligned}
$$

where $L$ is the Lagrangian function, and $\varphi$ is the Rayleigh dissipation function. Note that since the dependent variable in the MSD equations is the velocity, "energy" in this section refers to the energy associated with the change in this variable (i.e., associated with acceleration $u$ ). The "kinetic energy" here is thus $\dot{u}^{2} / 2$, rather than the physical kinetic energy $u^{2} / 2$, although the Lagrangian of the physical kinetic energy can also be easily derived. This Lagrangian is thus related (but not exactly equal) to the time rate of change of the physical kinetic energy. Using these Lagrangian functions, we may also derive the Hamiltonian (total energy $=$ kinetic + potential) of the EBL as

$$
\begin{aligned}
H_{u}[u(z), v(z), \dot{u}(z)]= & \frac{1}{2} \dot{u}(z)^{2}+\frac{1}{2} f^{2} u(z)^{2} \\
& +f \alpha(z) u(z) v(z) \text { and } \\
H_{v}[v(z), u(z), \dot{v}(z)]= & \frac{1}{2} \dot{v}(z)^{2}+\frac{1}{2} f^{2} v(z)^{2}-f \alpha(z) v(z) u(z) .
\end{aligned}
$$

These equations confirm that $f \alpha(z) v(z)$ and $f \alpha(z) u(z)$, or the last terms on the RHS of Eqs. (15a), (15b), and (16), only redistribute energy between the components and that $u$ and $v$ constitute the reservoirs of potential energy for each other. This term is related to the Coriolis force that couples Eqs. (15a) and (15b). We can observe this more clearly if we sum $H_{u}$ and $H_{v}$ to find the Hamiltonian of the whole system:

$$
\begin{aligned}
H_{u+v}[u(z), v(z), \dot{u}(z), \dot{v}(z)]= & \frac{1}{2}\left[\dot{u}(z)^{2}+\dot{v}(z)^{2}\right] \\
& +\frac{1}{2} f^{2}\left[u(z)^{2}+v(z)^{2}\right] .
\end{aligned}
$$

The first term on the RHS of this equation is the kinetic energy, and the second term is the potential energy of each layer of the EBL. We can plot these quantities from our LES results. Figure 6 (left) depicts the Hamiltonian function in the $u$ direction at two heights for case 3 (along with $u$ itself). As expected, the Hamiltonian varies with $\dot{u}$ and thus increases when the flow is accelerating and decreases when it is decelerating. Moreover, as height increases, the energy of the system also increases. Another insight that can be gained from the Hamiltonian is that, since this case involves resonance, its total energy increases continuously as one could observe from Fig. 6 (right). If the system was undamped (e.g., inviscid flow), its total energy would increase indefinitely; however, since the EBL includes the damping effects, this total energy will converge to a bounded value. Resonance is related to energy input by the forcing that is faster than the system's ability to dissipate.

\section{b. Resonance transmissibility and the damping ratio of the EBL}

The damped resonant frequency, which is the frequency at which the maximum gain or transmissibility (defined as output/input of energy) of the MSD system occurs, is different from the damped frequency. According to Eq. (17), the damped frequency of the EBL equals the Coriolis parameter; this is the frequency at which an unforced system released from an initial nonequilibrium position will oscillate. On the other hand, the damped resonant frequency for the EBL MSD system can be calculated as $\omega_{\text {peak }}=\omega_{n} \sqrt{1-2 \zeta^{2}}=\sqrt{f^{2}-\alpha^{2}}$. For our studied cases, $\alpha$ is approximately one order of magnitude smaller than $f$ (weakly damped system), and hence the damped resonant frequency is almost equal to the damped frequency of the EBL (since $\alpha^{2}$ is two orders smaller than $f^{2}$ ). This explains why the forcing at the Coriolis frequency (the damped frequency for our system) resulted in strong resonance.

One may also compute the damping ratio of this coupled MSD system based on Eq. (16) as $\zeta=|\alpha /[2 \sqrt{f(f-i \alpha)}]|$ to assess how far the system is underdamped. If the turbulence of the system at any particular height or time becomes large enough, the state of the system could switch from underdamped to critically damped or overdamped. One can already observe that, if $f$ is too small (as one approaches the equator), the damping ratio will increase, the system will invariably become overdamped, and inertial oscillation in the EBL are unlikely (though other restoring forces can create other types of inertial oscillations); our model and analyses thus do not apply near the equator. In our simulations, the damping ratio roughly varied between 0.012 and 0.16 .

\section{Conclusions}

This study numerically simulated the unsteady EBL and proposed a reduced physical model to characterize its dynamics. Five large-eddy simulations were executed to illustrate that the EBL behaves very much like an underdamped mass-spring-damper system (or, more generically, any underdamped harmonic oscillator) and 

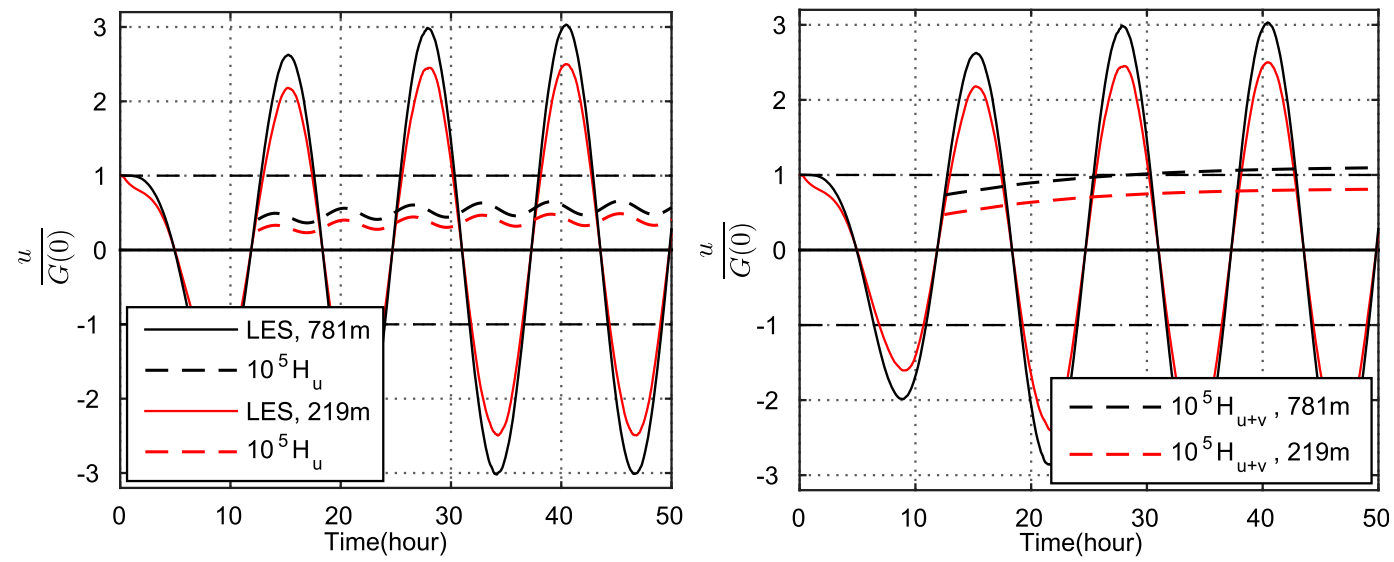

FIG. 6. Times series of (left) $u$ (solid lines) and $H_{u}$ (dashed lines) and (right) $u$ (solid lines) and $H_{u+v}$ (dashed lines) for simulated case 3 .

to validate the reduced model. When the forcing is very fast compared to EBL response time, the EBL cannot adjust itself to the exerted forcing, whereas it responds fully to a very slow sinusoidal forcing. The velocity profiles are distinct from the steady cases and do not necessarily feature a clear Ekman spiral or a supergeostrophic maximum (a supplemental file with animations showing the time evolution of the profiles is available online).

The analytical model for the unsteady EBL was derived from the RANS equations. The stress divergence was parameterized using a closure for the second gradient of the velocity, yielding a simplification of the PDEs into two coupled linear ODEs, each of which is analogous to an MSD system. The analytical solutions of these coupled ODEs require only one tunable parameter that is associated with the turbulent viscosity. If a reliable turbulent viscosity model is available, the MSD system will be closed and solvable. Here, we proposed a closure [Eq. (21)] for neutral conditions; only the forcing function is then needed to predict the velocity time series. Unstable or stable conditions can be readily modeled by simply deriving an appropriate relation between $\alpha$ and $\nu$ (potentially based on the Monin-Obukhov similarity theory) and adjusting the turbulent viscosity parameterization to account for the effect of buoyancy.

The analytical model was validated using LES in two modes: a "hindcasting" mode, where the tunable coefficient is directly optimized to match LES solutions (OMSD), and a "forecasting" mode, where a parameterization for the dependence of the turbulent viscosity on geostrophic forcing and height [Eq. (21)] is used (PMSD). In three test cases, the OMSD matches LES results remarkably well, confirming that the structure of the model and its assumptions are valid. The PMSD also performs well, but a more accurate eddy-viscosity model, which is not the focus of this paper, is needed for its performance to match the LES as well as the OMSD. Particularly, relaxation of the Boussinesq analogy assumption would significantly improve the performance of the PMSD model. Of course, real-world boundary layer dynamics result from many forces and physical processes, with various temporal and spatial scales, that combine to determine the time variation of atmospheric or oceanic boundary layer flows. Here, we have only focused on time variations of the pressure gradients, but the framework can be generalized to include other sources of unsteadiness, such as surface heat fluxes [via a time-varying turbulent viscosity parameterization $\alpha(t)$ ].

With PMSD, one can predict short-term variations of the mean wind velocities employing a turbulent viscosity parameterization and the suggested MSD model without requiring LES or any other numerical simulations. This is the novelty of this model, which can, for example, be applied in the atmospheric boundary layer for short-term forecasts of the wind variability that are critical to predict wind power production changes (Zhu and Genton 2012). This mass-spring-damper model of the EBL explains the variability of the unsteady flow in a more straightforward and understandable way compared to more complicated solutions of the original PDEs, as illustrated, for example, in the last section thorough the Lagrangian and Hamiltonian energy analyses that elucidated the origins of kinetic and potential energy. Compared to previous approaches, this new model can be solved for a wide range of viscosity and forcing (pressure gradient) variabilities in the EBL as a combined function of height and 

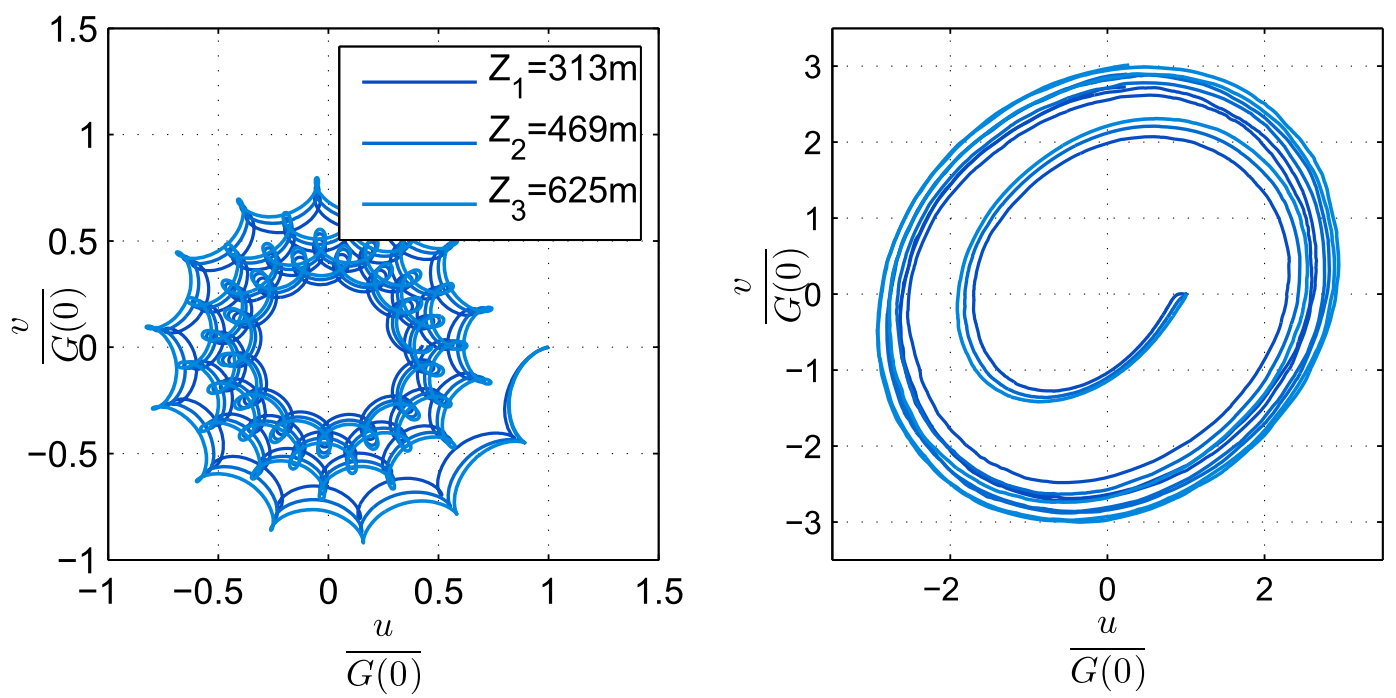

FIG. A1. The $u-v$ hodographs for two frequencies of forcing corresponding to cases (left) 1 and (right) 3 .

time and can thus have a wide range of practical applications.

Acknowledgments. The authors acknowledge support from the Cooperative Institute for Climate Science (CICS) of Princeton University and the National Oceanographic and Atmospheric Administration (NOAA) under Grant 344-6127, as well as the Physical and Dynamic Meteorology Program of the National Science Foundation under AGS-1026636. The simulations were performed on the computing clusters of the National Center for Atmospheric Research under Project P36861020.

\section{APPENDIX A \\ $u-v$ Hodographs}

The $u-v$ hodographs for cases 1 and 3 are shown in Fig. A1. Starting from point $(u=1, v=0)$ as the initial condition, $u$ and $v$ are evolved over $50 \mathrm{~h}$, and the general and particular behavior of the EBL can be observed in Fig. A1. These hodographs demonstrate how the forcing frequency and type generate distinct $u-v$ structures and patterns and illustrate that the EBL response is highly dependent on the forcing variability regime.

\section{APPENDIX B}

\section{Mathematical Derivation of Eq. (17)}

The full homogenous solution of Eq. (16) could be found as

$$
A_{h}(t)=C_{1} e^{-\alpha t}\left(\cos f_{c} t-i \sin f_{c} t\right)+C_{2}\left(\cos f_{c} t+i \sin f_{c} t\right) .
$$

Here, we prove that if one inserts the initial conditions and particular solution of our MSD model of Eq. (16) back into the original RANS equations, the undampedoscillator coefficient will be identically zero. We start with Eq. (16) and define the initial conditions of the full and the particular solution as

$$
\begin{aligned}
A_{0} & \equiv A(t=0)=u_{0}+i v_{0}, \\
A_{1} & \equiv \frac{d A}{d t}(t=0)=\left.\frac{d}{d t}(u+i v)\right|_{t=0} \quad \text { and } \\
A_{p_{0}} & \equiv A_{p}(t=0), \quad A_{p_{1}} \equiv \frac{d A_{p}}{d t}(t=0) .
\end{aligned}
$$

Using Eq. (B1) and writing the full solution, one obtains

$$
\begin{aligned}
A_{0} & =C_{1}+C_{2}+A_{p_{0}} \text { and } \\
A_{1} & =(\alpha+i f)\left(C_{2}-A_{0}+A_{p_{0}}\right)+i f C_{2}+A_{p_{1}} \\
& =(\alpha+i 2 f) C_{2}+\left(A_{p_{0}}-A_{0}\right)(\alpha+i f)+A_{p_{1}} .
\end{aligned}
$$

These initial conditions must also satisfy the original RANS equations [Eq. (10)] so we can write it at initial time as

$$
\begin{aligned}
A_{1} & =f v_{0}-\alpha u_{0}-f V_{g_{0}}+i\left(f U_{g_{0}}-f u_{0}-\alpha v_{0}\right) \\
& =i f U_{g_{0}}-f V_{g_{0}}-(\alpha+i f) A_{0} .
\end{aligned}
$$

Now, substitute $A_{1}$ from Eq. (B4) into the RHS of (B3) and solve to get the following: 


$$
C_{2}=-\frac{(\alpha+i f) A_{p_{0}}+A_{p_{1}}+f V_{g_{o}}-i f U_{g_{0}}}{(\alpha+i 2 f)} .
$$

We should note that the particular solution must also satisfy the original RANS equation [Eq. (10)]; hence,

$$
A_{p_{1}}=i f U_{g_{0}}-f V_{g_{0}}-(\alpha+i f) A_{p_{0}} .
$$

Substituting Eq. (B6) into Eq. (B5) yields that $C_{2}$ is identically zero, and thus one obtains Eq. (17) in the paper.

\section{REFERENCES}

Baker, W. E., S. C. Bloom, J. S. Woolen, M. S. Nestler, E. Brin, T. Schaltter, and G. Branstator, 1987: Experiments with a three-dimensional statistical objective analysis scheme using FGGE data. Mon. Wea. Rev., 115, 272-296, doi:10.1175/ 1520-0493(1987)115<0272:EWATDS > 2.0.CO;2.

Berger, B. W., and B. Grisogono, 1998: The baroclinic, variable eddy viscosity Ekman layer: An approximate analytical solution. Bound.-Layer Meteor., 87, 363-380, doi:10.1023/ A:1001076030166.

Blackadar, A. K., 1957: Boundary layer wind maxima and their significance for the growth of nocturnal inversions. Bull. Amer. Meteor. Soc., 38, 283-290.

Bonner, W., and J. Paegle, 1970: Diurnal variations in boundary layer winds over the south-central United States in summer. Mon. Wea. Rev., 98, 735-744, doi:10.1175/1520-0493(1970)098<0735: DVIBLW $>2.3 . \mathrm{CO} ; 2$.

Bou-Zeid, E., C. Meneveau, and M. Parlange, 2005: A scaledependent Lagrangian dynamic model for large eddy simulation of complex turbulent flows. Phys. Fluids, 17, 025105, doi:10.1063/1.1839152.

Caldwell, D. R., C. W. Van Atta, and K. N. Helland, 1972: A laboratory study of the turbulent Ekman layer. Geophys. Fluid Dyn., 3, 125-160, doi:10.1080/03091927208236078.

Dellar, P. J., and R. Salmon, 2005: Shallow water equations with a complete Coriolis force and topography. Phys. Fluids, 17, 106601, doi:10.1063/1.2116747.

Du, Y., and R. Rotunno, 2014: A simple analytical model of the nocturnal low-level jet over the Great Plains of the United States. J. Atmos. Sci., 71, 3674-3683, doi:10.1175/ JAS-D-14-0060.1.

— — - and Q. Zhang, 2015: Analysis of WRF-simulated diurnal boundary layer winds in eastern China using a simple 1D model. J. Atmos. Sci., 72, 714-727, doi:10.1175/JAS-D-14-0186.1.

Durran, D. R., 1989: Improving the anelastic approximation. J. Atmos. Sci. 46, 1453-1461, doi:10.1175/1520-0469(1989)046<1453: ITAA $>2.0 . \mathrm{CO} ; 2$.

Ekman, V. W., 1905: On the influence of the Earth's rotation on ocean-currents. Ark. Mat. Astron. Fys., 2, 1-52.

Fleagle, R. G., and J. A. Businger, 1981: An Introduction to Atmospheric Physics. 2nd ed. International Geophysics Series, Vol. 25, Academic Press, 42 pp.

Fritts, D. C., and G. D. Nastrom, 1992: Sources of mesoscale variability of gravity waves. Part II: Frontal, convective, and jet stream excitation. J. Atmos. Sci., 49, 111-127, doi:10.1175/ 1520-0469(1992)049<0111:SOMVOG >2.0.CO;2.

Gayen, B., S. Sarkar, and J. R. Taylor, 2010: Large eddy simulation of a stratified boundary layer under an oscillatory current. J. Fluid Mech., 643, 233-266, doi:10.1017/S002211200999200X.
Grisogono, B., 1995: A generalized Ekman layer profile with gradually varying eddy diffusivities. Quart. J. Roy. Meteor. Soc., 121, 445-453, doi:10.1002/qj.49712152211.

Haurwitz, B., 1947: Comments on the sea-breeze circulation. J. Meteor., 4, 1-8, doi:10.1175/1520-0469(1947)004<0001: COTSBC $>2.0 . \mathrm{CO} ; 2$.

Hering, W., and T. Borden, 1962: Diurnal variations in the summer wind field over the central United States. J. Atmos. Sci., 19, 81-86, doi:10.1175/1520-0469(1962)019<0081: DVITSW $>2.0 . \mathrm{CO} ; 2$.

Herrmann, M. J., and O. S. Madsen, 2007: Effect of stratification due to suspended sand on velocity and concentration distribution in unidirectional flows. J. Geophys. Res., 112, C02006, doi:10.1029/2006JC003569.

Hoskins, B. J., 1975: The geostrophic momentum approximation and the semi-geostrophic equations. J. Atmos. Sci., 32, 233-242, doi:10.1175/1520-0469(1975)032<0233:TGMAAT>2.0.CO;2.

Hsu, B. C., X. Lu, and M. Kwan, 2000: LES and RANS studies of oscillating flows over flat plate. J. Eng. Mech., 126, 186-193, doi:10.1061/(ASCE)0733-9399(2000)126:2(186).

Lentz, S. J., 2001: The influence of stratification on the wind-driven cross-shelf circulation over the North Carolina shelf. J. Phys. Oceanogr., 31, 2749-2760, doi:10.1175/1520-0485(2001)031<2749: TIOSOT $>2.0 . \mathrm{CO} ; 2$.

Lewis, D. M., and S. E. Belcher, 2004: Time-dependent, coupled, Ekman boundary layer solutions incorporating Stokes drift. Dyn. Atmos. Oceans, 37, 313-351, doi:10.1016/ j.dynatmoce.2003.11.001.

Lohmann, I. P., J. Fredsøe, B. M. Sumer, and E. D. Christensen, 2006: Large eddy simulation of the ventilated wave boundary layer. J. Geophys. Res., 111, C06036, doi:10.1029/ 2005JC002946.

Martlatt, S. W., S. B. Waggy, and S. Biringen, 2010: Direct numerical simulation of the turbulent Ekman layer: Turbulent energy budgets. J. Thermophys. Heat Transfer, 24, 544-555, doi:10.2514/1.45200.

Miles, J., 1994: Analytical solutions for the Ekman layer. Bound.Layer Meteor., 67, 1-10, doi:10.1007/BF00705505.

Nastrom, G. D., and D. C. Fritts, 1992: Sources of mesoscale variability of gravity waves. Part I: Topographic excitation. J. Atmos. Sci., 49, 101-110, doi:10.1175/1520-0469(1992)049<0101: SOMVOG $>2.0 . \mathrm{CO} ; 2$.

Parmhed, O., I. Kos, and B. Grisogono, 2005: An improved Ekman layer approximation for smooth eddy diffusivity profiles. Bound.Layer Meteor., 115, 399-407, doi:10.1007/s10546-004-5940-0.

Polavarapu, S. M., 1995: Divergent wind analyses in the oceanic boundary layer. Tellus, 47A, 221-239, doi:10.1034/ j.1600-0870.1995.t01-1-00005.x.

Pollard, R. T., and R. C. Millard, 1970: Comparison between observed and simulated wind-generated inertial oscillations. Deep-Sea Res. Oceanogr. Abstr., 17, 813-821, doi:10.1016/ 0011-7471(70)90043-4.

Pope, S. B., 2000: Turbulent Flows. 1st ed. Cambridge University Press, 802 pp.

Prandle, D., 1982: The vertical structure of tidal currents and other oscillatory flows. Cont. Shelf Res., 1, 191-207, doi:10.1016/ 0278-4343(82)90004-8.

$\mathrm{Pu}, \mathrm{B}$., and R. E. Dickinson, 2014: Diurnal spatial variability of Great Plains summer precipitation related to the dynamics of the low-level jet. J. Atmos. Sci., 71, 1807-1817, doi:10.1175/ JAS-D-13-0243.1.

Salmon, R., 1983: Practical use of Hamilton's principle. J. Fluid Mech., 132, 431-444, doi:10.1017/S0022112083001706. 
Schmidt, F., 1947: An elementary theory of the land- and seabreeze circulation. J. Meteor., 4, 9-20, doi:10.1175/ 1520-0469(1947)004<0009:AETOTL>2.0.CO;2.

Shapiro, A., and E. Fedorovich, 2010: Analytical description of a nocturnal low-level jet. Quart. J. Roy. Meteor. Soc., 136, 12551262, doi:10.1002/qj.628.

Shingai, K., and H. Kawamura, 2004: A study of turbulence structure and large-scale motion in the Ekman layer through direct numerical simulations. J. Turbul., 5, 37-41, doi:10.1088/ 1468-5248/5/1/013.

Sitaraman, V., and V. V. Shirvaikar, 1974: Lagrangian similarity theory applied to diffusion in the planetary boundary layer. Bound.-Layer Meteor., 5, 419-427, doi:10.1007/ BF00123489.

Sous, D., J. Sommeria, and D. Boyer, 2013: Friction law and turbulent properties in a laboratory Ekman boundary layer. Phys. Fluids, 25, 046602, doi:10.1063/1.4802045.

Spalart, P. R., G. N. Coleman, and R. Johnstone, 2008: Direct numerical simulation of the Ekman layer: A step in Reynolds number, and cautious support for a log law with a shifted origin. Phys. Fluids, 20, 101507, doi:10.1063/1.3005858.
Stull, R., 1988: An Introduction to Boundary Layer Meteorology. 1st ed. Atmospheric and Oceanographic Sciences Library, Vol. 13, Kluwer Academic Publishers, 670 pp.

Tan, Z. M., 2001: An approximate analytical solution for the baroclinic and variable eddy diffusivity semi-geostrophic Ekman boundary layer. Bound.-Layer Meteor., 98, 361-385, doi:10.1023/ A:1018708726112.

Tennekes, H., and J. Lumley, 1972: A First Course in Turbulence. MIT Press, $300 \mathrm{pp}$.

Vallis, G. K., 2006: Atmospheric and Oceanic Fluid Dynamics. Cambridge University Press, 745 pp.

Van de Wiel, B. J. H., A. F. Moene, G. J. Steeneveld, P. Baas, F. C Bosveld, and M. Holtslag, 2010: A conceptual view on inertial oscillations and nocturnal low-level jets. J. Atmos. Sci., 67, 2679-2689, doi:10.1175/2010JAS3289.1.

Veronis, B. G., 1967: Analogous behavior of homogeneous, rotating fluids and stratified, non-rotating fluids. Tellus, 19A, 326-336, doi:10.1111/j.2153-3490.1967.tb01485.x.

Zhu, X., and M. G. Genton, 2012: Short-term wind speed forecasting for power system operations. Int. Stat. Rev., 80, 2-23, doi:10.1111/j.1751-5823.2011.00168.x. 


\title{
CORRIGENDUM
}

\author{
MOSTAFA MOMEN \\ Department of Earth System Science, Stanford University, Stanford, California, and Department of Civil and Environmental \\ Engineering, Princeton University, Princeton, New Jersey \\ ELIE BOU-ZEID \\ Department of Civil and Environmental Engineering, Princeton University, Princeton, New Jersey
}

(Manuscript received 26 July 2017, in final form 14 August 2017)

In Momen and Bou-Zeid (2016, p. 30), the second term on the right-hand side of Eq. (10) has a sign error and must be corrected as follows:

$$
\begin{aligned}
& \frac{\partial u}{\partial t}=-f_{c}\left(V_{g}-v\right)-\frac{\partial}{\partial z}\left(\overline{u^{\prime} w^{\prime}}\right), \\
& \frac{\partial v}{\partial t}=f_{c}\left(U_{g}-u\right)-\frac{\partial}{\partial z}\left(\overline{v^{\prime} w^{\prime}}\right) .
\end{aligned}
$$

Following this change, Eq. (11) also requires a modification as

$$
\begin{aligned}
& -\frac{\partial}{\partial z}\left(\overline{u^{\prime} w^{\prime}}\right) \simeq \frac{\partial}{\partial z}\left(\nu_{T} \frac{\partial u}{\partial z}\right)=\frac{\partial \nu_{T}}{\partial z} \frac{\partial u}{\partial z}+\nu_{T} \frac{\partial^{2} u}{\partial z^{2}}=-\alpha(z, t) u(z, t), \\
& -\frac{\partial}{\partial z}\left(\overline{v^{\prime} w^{\prime}}\right) \simeq \frac{\partial}{\partial z}\left(\nu_{T} \frac{\partial v}{\partial z}\right)=\frac{\partial \nu_{T}}{\partial z} \frac{\partial v}{\partial z}+\nu_{T} \frac{\partial^{2} v}{\partial z^{2}}=-\alpha(z, t) v(z, t) .
\end{aligned}
$$

The two sign changes cancel out and the subsequent equations and results remain unchanged.

\section{REFERENCE}

Momen, M., and E. Bou-Zeid, 2016: Large-eddy simulations and damped-oscillator models of the unsteady Ekman boundary layer. J. Atmos. Sci., 73, 25-40, doi: 10.1175/JAS-D-15-0038.1. 